\title{
Metro Vehicle Axle Temperature Monitoring Device Based on Piezoelectric Vibration
}

\author{
Yiyun Zhao*, Ruyan Huang \\ College of Urban Railway Transportation, Shanghai University of Engineering Science, Shanghai, China \\ Email address: \\ 506504590@qq.com (Yiyun Zhao), sues101114137@163.com (Ruyan Huang) \\ *Corresponding author
}

\section{To cite this article:}

Yiyun Zhao, Ruyan Huang. Metro Vehicle Axle Temperature Monitoring Device Based on Piezoelectric Vibration. International Journal of Transportation Engineering and Technology. Vol. 5, No. 4, 2019, pp. 88-91. doi: 10.11648/j.ijtet.20190504.14

Received: November 2, 2019; Accepted: December 3, 2019; Published: December 9, 2019

\begin{abstract}
The axle temperature monitoring of subway vehicles is a key technology to ensure the safety of trains. The existing axle temperature monitoring system is monitored by using trackside equipment or using axle temperature test paper, the system has the inferiority of poor real-time performance. In order to realize real-time and online monitoring of axle temperature, this paper presents a subway axle temperature monitoring device based on vibration energy recovery. The device is installed on the shaft end of the train axle, and uses the positive piezoelectric effect of piezoelectric ceramics to absorb vibration energy into electric energy, and continuously sends temperature signal through the radio frequency module to realize real-time monitoring of train axle temperature. A mathematical model of piezoelectric vibrator power output was established to obtain design parameters suitable for rail vehicle vibration, and the Ansys Workbench finite element analysis software was used for theoretical simulation verification. Finally, an experimental model was built and a vibration test was carried out on Shanghai metro line 1. The results show that the axial temperature monitoring device based on piezoelectric vibration can effectively obtain the axial temperature data and realize the online monitoring function.
\end{abstract}

Keywords: Axial Temperature Monitoring, Energy Recovery, Piezoelectric Ceramics, Finite Element Analysis

\section{Introduction}

With the continuous increase of train speed and load, the occurrence of hot shaft, cutting shaft and even burning shaft accidents intensification, wheelset malfunction increasing obviously, especially rolling bearing malfunction [1]. Therefore, how to monitor the train running condition in real time and predict the axle and bearing malfunction accurately has become an urgent question. Through monitoring the temperature of the vehicle axle, it is found that preventing the hot axle and burning axle of the vehicle is an important measure to ensure the safety of the train [2]. At present, the widely used monitoring methods of train axle temperature can be divided into two types: vehicle contact and rail side induction [3-7]. Due to the influence of external environment and the difficulty of positioning, the rail side induction system has the disadvantages of low alarm accuracy, high false alarm rate and high cost. At present, most vehicle-mounted contact devices adopt the wireless network based on ZigBee technology to monitor data in real time [8-10]. Because ZigBee technology is a close transmission communication technology, data transmission between locomotive monitoring platform and station control room platform cannot be realized at the same time. Besides, these sensors and radio frequency modules all need external power supply, and the use of power supply in the carriage will easily damage the layout structure of the carriage, bringing unexpected safety risks. An energy recovery technology based on piezoelectric effect has been applied in many fields, which provides a new method for power supply of train axle temperature sensor. [11-15]

\section{Main Body}

\subsection{System Scheme and Framework}

According to the actual situation and requirements of the train monitoring system, we designed the train malfunction monitoring system in the figure. Mainly used for axle malfunction monitoring of freight trains. Firstly, the energy 
generated by the piezoelectric vibrator passes through the conversion circuit to provide electrical energy for the whole system. Temperature signal is collected by temperature sensor, and the acceleration signal generated by the vibration of piezoelectric vibrator is obtained by ADC conversion circuit. The sampling sensor collects data and sends it to the main controller. The main controller reduces the amount of data sent by compression coding algorithm, and sends the data to the upper computer through NB-IoT and upload it to the database at the same time. After receiving the data, the upper computer uses the axle malfunction identification algorithm and displays the malfunction on the upper computer's display screen. Terminal device is the same, with different serial Numbers, to form a wireless network transmission system, so that the monitoring platform of the locomotive and the total monitoring platform of the station can obtain data at the same time, so as to achieve the monitoring purpose.

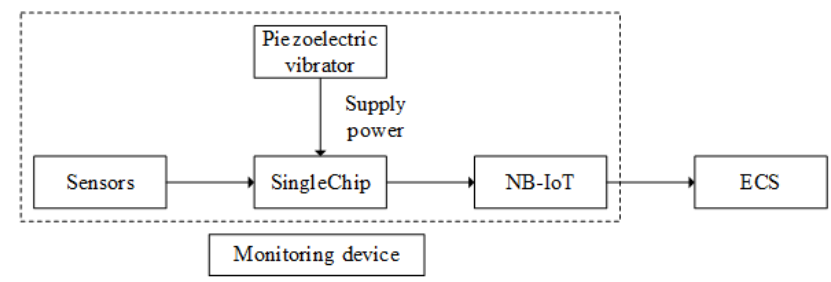

Figure 1. System structure diagram.

This system breaks through the external power supply mode of the traditional signal acquisition system. By using the vibration energy of the train, self-collection, self-power supply and self-management can be realized without external intervention. Each acquisition node devices have its own proprietary network to avoid channel conflicts and ensure real-time data. The three-level network structure is adopted to extend the coverage of the network and realize the omni-directional monitoring of the train. The dual sensor design ensures the reliability of data acquisition.

\subsection{Key Technology and Theoretical Analysis}

At present, the vibration of urban rail transit trains is characterized by sparse transverse vibration energy and intensive longitudinal vibration energy. According to this characteristic, a simple beam type circular piezoelectric vibrator is set up as the core structure of energy acquisition. The model is simplified as figure 1 . The three-layer structure is adopted. The upper and lower surfaces of the brass substrate are coaxial fixed piezoelectric materials, which can make the piezoelectric material bear less stress while obtaining more power generation. According to the research, the vibration frequency of the train is relatively low. In order to lower the natural frequency of the system, a mass block is fixed in the middle by anti-loosening bolt, and the shape variable of piezoelectric material can be increased to increase the power generation.

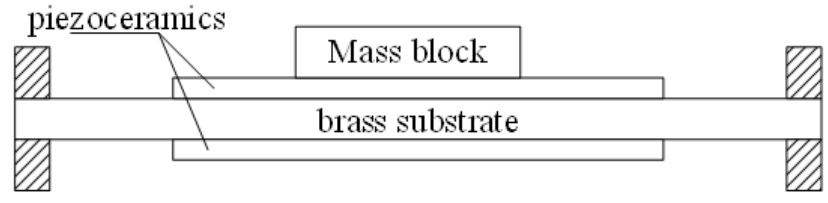

Figure 2. Piezoelectric vibrator equivalent model.

The device converts the collected energy into electricity supply appliances, and the equivalent circuit is as follows:

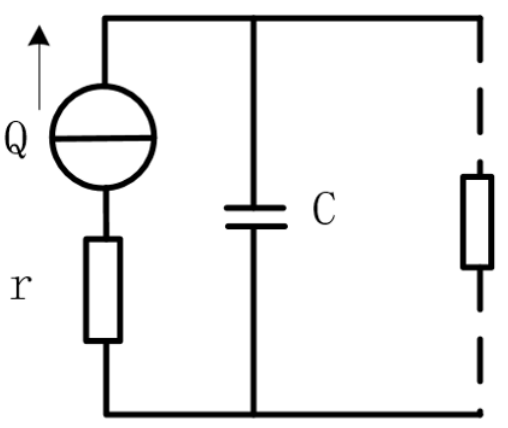

$\mathrm{R}$

Figure 3. Piezoelectric vibrator equivalent circuit.

Where, $\mathrm{Q}$ is the equivalent charge source of piezoceramics, $\mathrm{C}$ is the equivalent capacitance of piezoceramics, $\mathrm{r}$ is the internal resistance of the acquisition circuit, $\mathrm{R}$ refers to the external load, and the dotted line is the actual working situation. Here, the equivalent circuit without load is only discussed. Based on the above equivalent model and equivalent circuit, the mathematical model of piezoelectric vibrator power can be calculated:

$$
P=\frac{u n \omega d_{33}^{2} K^{2} \Delta x^{2} h^{2}}{\varepsilon^{2} S^{2} r}
$$

In the formula $1, \mathrm{n}$ is the number of piezoceramics, $\mathrm{u}$ is the number of a single cycle vibration, $\omega$ is the frequency of the vibration of the work environment, $\mathrm{d} 33$ is the piezoelectric strain constant, $\mathrm{K}$ is equivalent elastic coefficient of Piezoelectric vibrator, $\Delta \mathrm{x}$ is an effective form of piezoceramics variables, $h$ is the thickness of the piezoceramics, $\varepsilon$ is the permittivity of piezoceramics, $\mathrm{S}$ is the area of the piezoceramics.

Among them, the effective shape variable of piezoceramics can be calculated by the mechanical model. The figure below shows the equivalent force of piezoceramics:

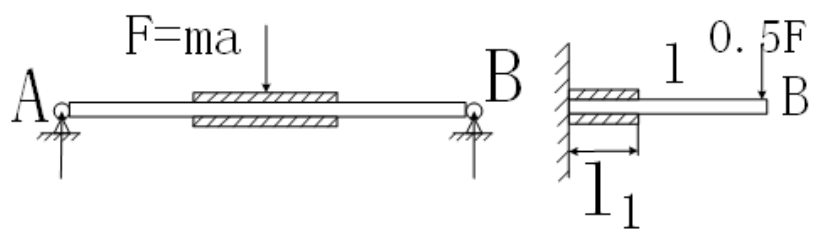

Figure 4. Piezoelectric vibrator equivalent force.

Because the model is symmetric, only one side needed to be calculated. Since the model can be regarded as a 
variable-section beam, it can be calculated and solved according to the calculation formula of the variable-section beam in material mechanics by equivalent toughening.

$$
\Delta x=\frac{\frac{1}{2} F l_{1}^{3}}{3 E_{1} I_{1}}+\frac{\frac{1}{2} F\left(l-l_{1}\right) l_{1}^{2}}{2 E_{1} I_{1}}+\left(\frac{\frac{1}{2} F l_{1}^{2}}{2 E_{1} I_{1}}+\frac{\frac{1}{2} F\left(l-l_{1}\right) l_{1}}{E_{1} I_{1}}\right)\left(l-l_{1}\right)+\frac{\frac{1}{2} F\left(l-l_{1}\right)^{3}}{3 E_{2} I_{2}}
$$

The moment of inertia of rectangular beam is:

$$
I_{1}=\frac{b h^{3}}{12}
$$

From the formula mentioned above, it can be seen that there are six influencing factors affecting the power generation of the system:

(1) mass, (2) acceleration (frequency included), (3) structure and constraint mode, (4) length, width and thickness of piezoceramics, (5) length, width and thickness of metal substrate, (6) power generation performance parameters of piezoceramics. By changing the above parameters, the piezoelectric vibrator can be optimized to enhance the power generation performance and structural reliability.

\subsection{Experiment and Simulation}

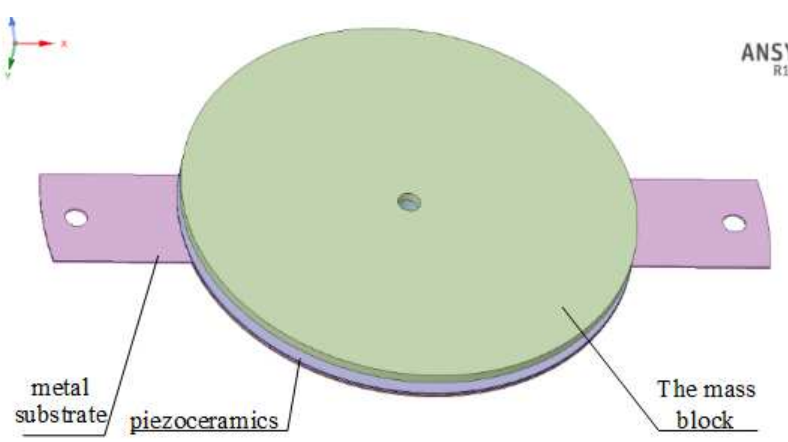

Figure 5. Three-dimensional model of piezoelectric vibrator.

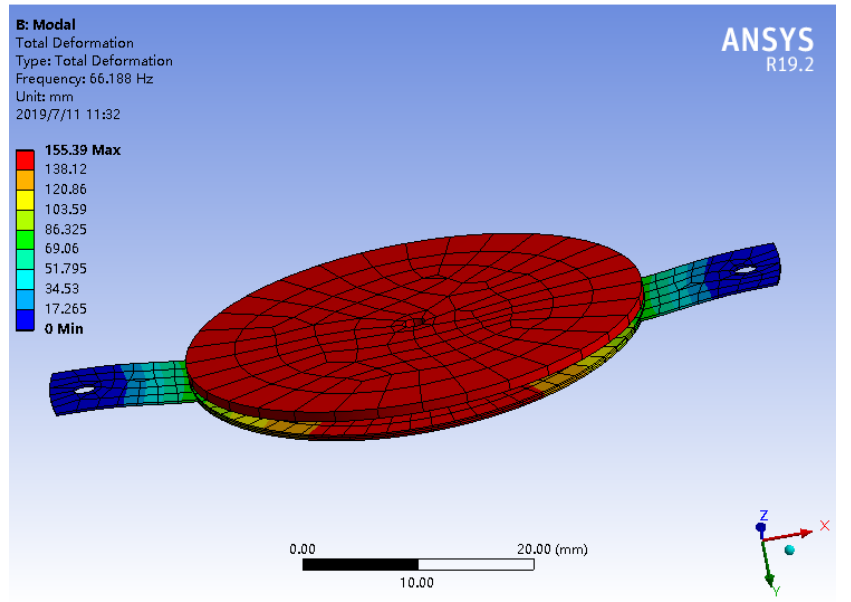

Figure 6. First modal shape of piezoelectric vibrator.

In order to study modal frequency characteristics of Piezoelectric vibrators and stress distribution in working environment, finite element analysis software based on Ansys Workbench was used for simulation. Spaceclaim was used to draw a three-dimensional model of piezoelectric vibrators, as shown in figure 5. Modal analysis was carried out on the model to obtain the first order modal shape and frequency of the Piezoelectric vibrators, as shown in figure 6.

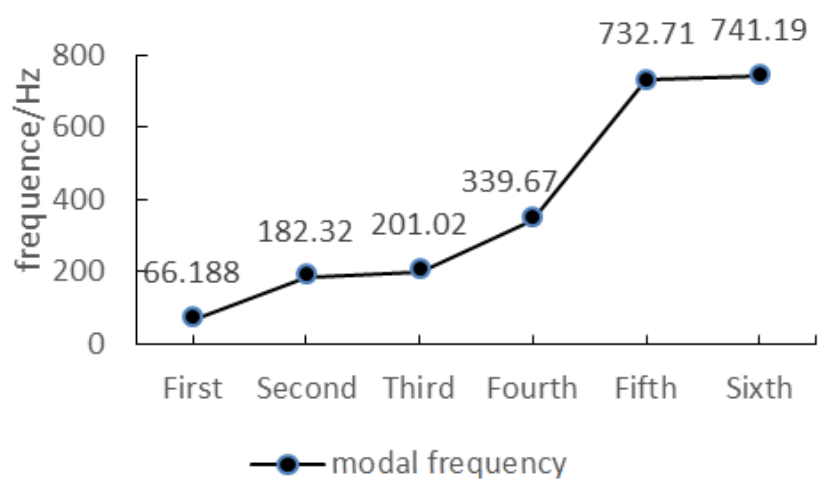

Figure 7. Modal frequency of piezoelectric vibrator.

The natural frequency of the piezoelectric vibrator is inversely proportional to the mass of the mass block. Experimental data show that in the actual working environment of Shanghai metro, the vibration energy is concentrated at the two frequencies of $75 \mathrm{~Hz}$ and $400 \mathrm{~Hz}$. In order to make the piezoelectric vibrator generate more power, it is necessary to make it resonate as much as possible, that is, to change the natural frequency by increasing or decreasing the mass block, so that it is close to the working frequency.

In order to ensure the reliability of the device, stress simulation is carried out on the piezoelectric vibrator, and the stress distribution diagram is shown in figure 8. According to the figure, under the simulation environment of $20 \mathrm{~g}$ acceleration load and $500 \mathrm{~Hz}$ frequency, the maximum stress of the piezoelectric vibrator is concentrated at the screw holes on both sides of the metal substrate. Among the components of piezoelectric vibrator, the most vulnerable component to failure is piezoelectric ceramics, whose surface stress is dispersed, enhancing the reliability of the whole device.

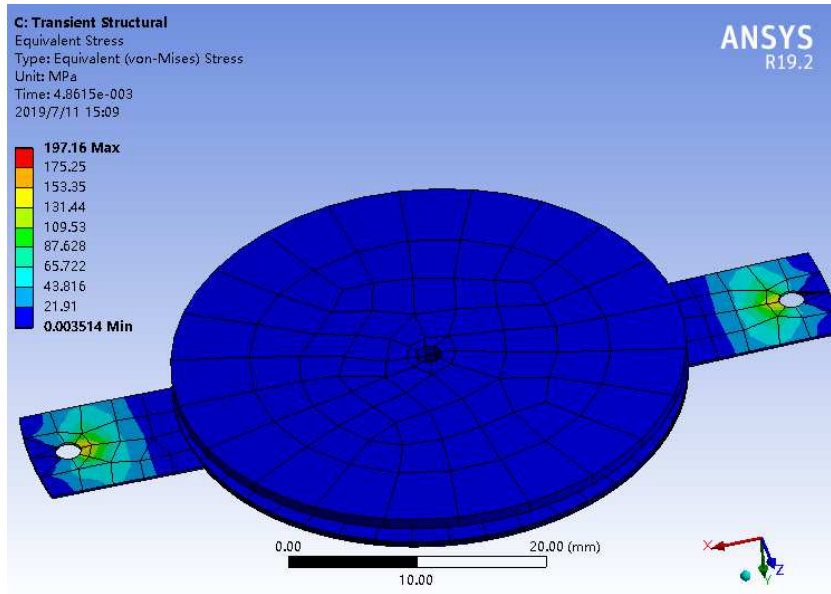

Figure 8. The stress distribution diagram of piezoelectric vibrator.

In order to prove the safety, reliability and vibration energy 
recovery performance of the device during train operation, we installed the device at the axle end of Shanghai metro line 1 train at the Meilong base of Shanghai rail transit, and carried out real subway environment experiment. Experimental data show that in the working environment of the train, the device completes the continuous transmission of temperature data under the condition of Self-generation electricity.

\section{Conclusion}

The theoretical power output model of the piezoelectric vibrator is established, and six influencing factors affecting the power generated by the piezoelectric ceramics are analyzed. It is known that the power generated by the piezoelectric ceramics is proportional to its effective shape variable in a certain range.

ANSYS was used to establish the finite element simulation model of piezoelectric ceramics, and the stress distribution of the device in the simulated working environment was analyzed. Under the simulation environment of $20 \mathrm{~g}$ acceleration load and $500 \mathrm{~Hz}$ frequency, the maximum stress of the device was $197.16 \mathrm{Mpa}$, which passed the reliability test.

\section{References}

[1] ZHOU S R, ZHONG S, ZHANG P. Analysis of research status on fault diagnosis of train roller bearing $[\mathrm{J}]$. China railway, 2009 (11): 35-39.

[2] HE C, YANG Q. Online detection system of wheelset vibration and axle temperature [J]. Electric locomotive and urban rail vehicle, 2018, 41 (04): 61-64.

[3] LI P. Research and design of standard emu axle temperature monitoring device [D]. Southwest jiaotong university, 2018.

[4] ZHANG R, ZHANG L L. Freight train axle temperature alarm and monitoring system based on LabVIEW [J]. Gansu science and technology, 2012, 28 (24): 46-48.

[5] LIU T. Research and design of high-speed railway axle temperature detection system [D]. Southwest jiaotong university, 2008.

[6] QIN Y M. Research on key technologies of train safety monitoring system [D]. Southwest jiaotong university, 2006.

[7] LIU X D, CHE H J. Master-slave shaft temperature test system [J]. Journal of qiqihar university, 2004 (03): 66-68.

[8] ZHANG J Z. Infrared monitoring technology and system application of train axle temperature [J]. Coal mine electromechanical, 2003 (01): 13-14+43.

[9] HUANG J, SHAN H. Zigbee-based wireless self-organizing network freight train axle temperature monitoring system [J]. Computer and information technology, 2006 (10): 82-85.

[10] XIE Q Y. Design and implementation of Zigbee-based subway train axle temperature detection [D]. Southwest jiaotong university, 2017

[11] TEKKALMAZ M, KORPEOGLU I. Distributed power source aware routing in wireless sensor networks [J]. Wireless Networks, 2016, 22 (4): 1381-1399.

[12] HU G, TSE K T, KWOK K CS, et al. Aerodynamic modification to a circular cylinder to enhance the piezoelectric wind energy harvesting [J]. Applied Physics Letters, 2016, 109 (19): 193902.

[13] REN L, CHEN R W, BURROW S, et al. Design and performance test of a high volumetric figure of merit electromagnetic vibration energy harvester [J]. Journal of Vibration and Shock, 2018. 37 (10): 102-109.

[14] GUO Q, ZHANG J Y, WANG Y, et al. Study on fabrication and properties for small low- frequency electromagnetic energy harvester [J]. Instrument Technique and Sensor, 2017 (1): 26-28.

[15] WONG V K, HO J H, CHAI A B. Performance of a piezoelectric energy harvester in actual rain [J]. Energy, 2017, 124: 364-371. 